\title{
Alternative Transcarotid Approach for Endovascular Treat- ment of Acute Ischemic Stroke Patients: A Case Series
}

\author{
Hanna Styczen, $\mathrm{MD}^{1}$, Daniel Behme, $\mathrm{MD}^{1}$, Amelie Carolina Hesse ${ }^{1}$, Marios Nikos Psychogios, $\mathrm{MD}^{1,2}$ \\ ${ }^{1}$ Institute for Diagnostic and Interventional Neuroradiology, University Medical Center Goettingen, Goettingen, Germany \\ ${ }^{2}$ Department of Neuroradiology, Clinic of Radiology, Neuroradiology and Nuclear Medicine, University Hospital Basel, Basel, Switzerland
}

\begin{abstract}
Purpose: Mechanical thrombectomy has become the standard of care for acute stroke caused by large vessel occlusion. As more patients are treated endovascularly, the number of older patients with tortuous vessels has risen. In these patients, catheterizing the internal carotid artery via a transfemoral approach can be very difficult or even impossible. Therefore, in selected patients, alternative strategies to the transfemoral approach have to be applied.
\end{abstract}

Materials and Methods: We report a case series of six patients undergoing mechanical thrombectomy via a combined transfemoral and transcarotid approach. Puncture of the carotid artery was conducted using roadmap guidance after an unsuccessful transfemoral attempt. Technical aspects and outcomes with this alternative approach were analyzed.

Results: Direct puncture of the carotid artery was achieved in five out of six patients (83\%). In three out of six patients (50\%), revascularization (modified Thrombolysis in Cerebral Infarction score $\geq 2$ b) was restored. No complications related to endovascular therapy were documented. One patient showed good neurological outcome (modified Rankin Scale [mRS] 5 at admission, mRS 1 at discharge).

Conclusion: A combined transfemoral/transcarotid approach can be an alternative vascular access in patients with problematic vessel anatomy.

Key Words: Acute ischemic stroke; Carotid artery; Direct carotid puncture; Mechanical thrombectomy; Transcarotid access

\section{INTRODUCTION}

In patients with acute ischemic stroke undergoing mechanical thrombectomy (MT), transfemoral access is routinely used. Alternative transbrachial and transradial approaches in elective diagnostic cerebral angiography, carotid stenting, or embolization of an intracranial aneurysm are described in the literature. ${ }^{1-3}$ These alternative vascular accesses are favored in patients with difficult anatomy such as elongated aortic arch and severe calcified, tortuous, or stenotic proximal cervical vasculature. However, treatment of acute stroke patients is based on swift and complete reperfusion. Prolonged groin to reperfusion time due to technical difficulties in catheterizing proximal supraaortic vessels leading to termination of MT are associated with higher severity of poststroke disability and reduced rate of functional independence. ${ }^{4-6} \mathrm{~A}$ transcarotid approach has been described in several case studies. ${ }^{7-9}$ Furthermore, di-

\section{Correspondence to: Hanna Styczen, MD \\ Department of Diagnostic and Inter- ventional Neuroradiology, University Medical Center Goettingen, Robert- Koch-Str. 40, Goettingen 37075 , Germany \\ Tel: +49-551-3966661 \\ Fax: +49-551-3912868 \\ E-mail: hanna.styczen@med. \\ uni-goettingen.de}

Received: April 23, 2019

Revised: May 28, 2019

Accepted: June 21, 2019
Copyright $\odot 2019$ Korean Society of Interventional Neuroradiology This is an Open Access article distributed under the terms of the Creative Commons Attribution Non-Commercial License (http://creativecommons.org/licenses/by-nc/3.0) which permits unrestricted non-commercial use, distribution, and reproduction in any medium, provided the original work is properly cited.

pISSN 2093-9043 eISSN 2233-6273 
rect transcervical access and additional flow reversal for cerebral protection in patients undergoing carotid angioplasty and stenting was shown to be a safe technique for treating carotid stenosis in patients aged $>70$ years. ${ }^{10}$ Alvarez et al. ${ }^{10}$ concluded that avoiding the aortic arch and tortuous supra-aortic vessels was responsible for the favourable results in their study. Potential risks compared to the standard transfemoral access include more dire consequences in case of injury to the carotid artery and impeded navigation among other proximal cervical vessels in the event of intra-arterial complications.

Common approaches to puncturing the carotid artery involve ultrasound guidance or an open surgical access. ${ }^{7,8}$ In our study, we present an alternative method for direct puncture of carotid arteries using road map guidance with a diagnostic catheter placed in the ostium of the common carotid via a transfemoral approach in cases where the internal carotid could not be accessed with an $8 \mathrm{~F}$ catheter/sheath in the standard pathway.

\section{MATERIALS AND METHODS}

We report a retrospective analysis of all patient who were treated endovascularly via a combined transfemoral/transcarotid approach for large vessel occlusion in the anterior circulation between September 2017 and October 2018. Patient characteristics, radiological features, endovascular procedure details, complications, and angiographic and clinical outcomes were analyzed. Reperfusion results were reported according the modified Thrombolysis in Cerebral Infarction (mTICl) score. Clinical outcome was assessed with the National Institutes of Health Stroke Scale (NIHSS) and modified Rankin Scale (mRS) at discharge and follow-up. The need for informed consent for this study was waived due to the retrospective nature of this study. In the context of required emergency treatment, verbal consent was obtained whenever possible for MT and specifically for the transcarotid approach. Data were derived from a prospectively acquired database, which was approved by the Local Ethics Committee.

\section{Technical aspects}

In our department, MT is usually performed with an $8 \mathrm{~F}$ system, starting with a transfemoral approach. A short $8 \mathrm{~F}$ sheath was placed in the femoral artery, and we navigated towards the aortic arch and carotid arteries usually with an 8 F guide catheter (usually an 8 F VISTA Brite Tip; Johnson \& Johnson, New Brunswick, NJ, USA). In all six patients, catheterization of the common carotid arteries with the guide catheter was unsuccessful due to the anatomy of the aortic arch and the cervical vessels. Therefore, a long 5 F SIM 2 catheter was used in a triaxial approach with the help of a $0.035^{\prime \prime}$ wire for catheterization of the common and internal carotid arteries. In cases of unsuccessful navigation where neither the triaxial approach nor switching to a stiffer wire was effective, the guide catheter was removed and the ostium of the common carotid artery was reached with a $5 \mathrm{~F}$ SIM 2 catheter. A biplane roadmap of the cervical carotids was acquired after injection through a SIM 2 catheter (Fig. 1A, C). Since the fluoroscopic roadmap was motion sensitive, prior intubation of the patients was required. An $18 \mathrm{G}$ needle was placed cranially to the clavicle bone and navigated in a $45^{\circ}$ angle toward the common carotid artery on the biplane roadmap (Fig. 1B, D). After puncturing the artery, a 0.025" wire was advanced towards the internal carotid artery; and after removal of the needle, a $6 \mathrm{~F}$ flexible sheath (Glidesheath Slender; Terumo Medical, Somerset, NJ, USA) was placed inside the common carotid artery with the tip in the internal carotid artery. An aspiration catheter (Usually a CATALYST 6 F; Stryker Neurovascular, Fremont, CA, USA) was then advanced toward the distal segment of the internal carotid artery, and standard diagnostic subtraction angiography (DSA) was acquired. Mechanical thrombectomy was performed with a first-line use of a stent-retriever and aspiration catheter, as described in the SAVE-technique." After MT, the cervical puncture site was closed with the use of a closure device (usually ANGIO-SEAL; St. Jude Medical, St. Paul, MN, USA) and control DSA was performed with the maintained catheter in the proximal carotid artery placed via transfemoral access. Then, the femoral puncture site was also closed with the use of a closure device (usually ANGIO-SEAL; St. Jude Medical).

\section{RESULTS}

A total of six patients (four female) with a mean age of 79 years (range, 54-97) were included in this study (Table 1). Six patients in 247 overall thrombectomy procedures (2.4\%) underwent carotid access. Out of six patients, occlusion was located at the level of the Carotid T in two patients, three times in M1 segment and once in an M2 Segment. One pa- 
tient with carotid T thrombosis presented an additional highgrade ipsilateral stenosis of the internal carotid artery. Therefore, the patient first underwent angioplasty for the highgrade internal carotid stenosis. Then MT was performed.

Clinical symptoms included contralateral hemiparesis in six patients, dysarthria in three patients, global aphasia in two patients and neglect in two patients. The cause of ischemic stroke was cardiac embolism in three patients. One patient suffered from embolism due to severe arteriosclerosis of the carotid artery. In the remaining two patients, no identifiable cause of stroke was found. Three of six patients were
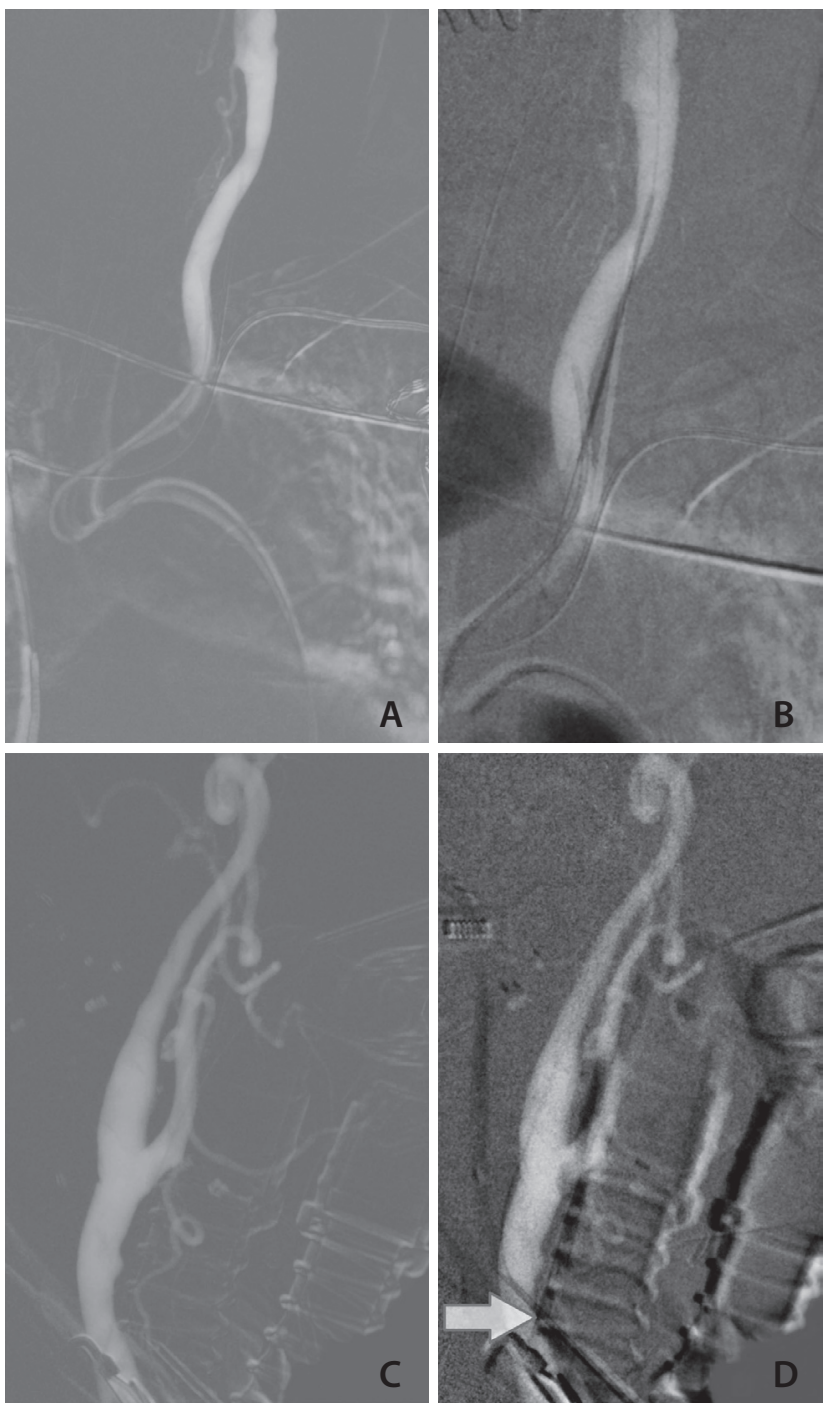

Fig. 1. Left common carotid angiogram showing a biplane roadmap after injection through the SIM 2 catheter placed via transfemoral access (A, antero-posterior view; $\mathbf{C}$, lateral view). An $18 \mathrm{G}$ needle (arrow) was placed cranially to the clavicle bone and navigated in a $45^{\circ}$ angle toward the common carotid artery on the biplane roadmap ( $\mathbf{B}$, antero-posterior view; D, lateral view).

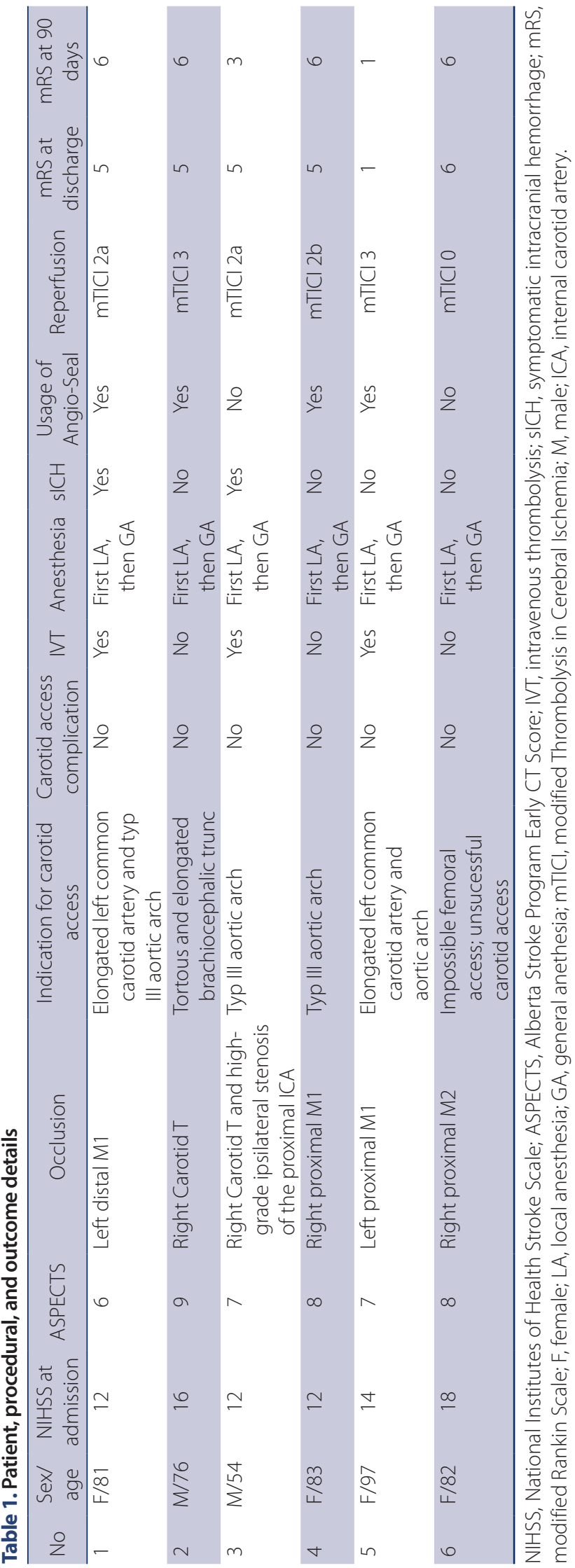


admitted as wake-up strokes with significant mismatch in cerebral blood volume and cerebral blood flow perfusion maps. In the remaining three patients, mean time from onset of symptoms to treatment was approximately 93 minutes (range, 83-110). Mean ASPECTS was 8 (range, 6-9). In five out of six patients, a triaxial approach with a guide catheter, a long 5 F SIM 2 catheter, and wire (including switching to stiffer or longer wires, exchange wire, different guides) was unsuccessful due to unfavorable aortic arch or vessel tortuosity. In one patient, femoral access was impossible and carotid access failed. Difficulty with cervical access in one case was due to the attempted access point being too proximal and close to the clavicle. The patient had a very short neck, and as a result there was difficulty advancing the sheath.

First the procedures were performed using only local anesthetic ( $1 \%$ lidocaine subcutaneously). Since the fluoroscopic roadmap is motion sensitive, prior intubation of the patients was required. Therefore, before puncture of the carotid artery, all patients received general anesthesia.

Successful reperfusion ( $\mathrm{mTICl} \geq 2 \mathrm{~b}$ ) was achieved in three out of six cases (50\%). Average groin to reperfusion time was 66 minutes (range, 54-86). Mean time from groin to carotid puncture and carotid puncture to reperfusion was 33 minutes (range, 20-46 and 15-50, respectively). Two patients suffered from symptomatic intracranial hemorrhage. In one patient, immediate post interventional flat detector computed tomography (CT) confirmed contralateral intracranial parenchymatous bleeding due to lysis therapy. Control CT in another patient showed secondary hematoma of the infarcted area and cerebral edema followed by decompressive hemicraniectomy. At discharge and after 90 days follow-up, one patient (age, 97) presented with progressive improvement neurologically with a mRS of 1 . The remaining four patients showed no difference in pre- and postinterventional neurological outcome (mRS 5). Mean pretreatment and posttreatment NIHSS was 13 (range, 12-18 and 2-26). One patient, whose endovascular intervention was terminated early, died of fulminant pulmonary embolism 17 days after admission. After 90 days of follow-up, four patients had died and two patients presented with a mRS of 1 and 3, respectively. In four patients, the puncture site was closed with ANGIO-SEAL (St. Jude Medical). Manual compression of the puncture site was sufficient for hemostasis in the remaining two cases. No complications (including relevant neck hematoma) related to endovascular therapy occurred.

\section{DISCUSSION}

There are limited studies with small patient collectives that analyze the transcarotid approach in patients undergoing endovascular treatment for acute ischemic stroke. ${ }^{7,12}$ In all published studies, first a transfemoral access was applied and puncture of the carotid artery was only contemplated if transfemoral catheterizing failed. Predictors associated with prolonged time to carotid access include age $>75$ years, dyslipidaemia, hypertension, and affection of the left carotid circulation. ${ }^{6}$ In our series, all aforementioned factors contributed to difficulty with the carotid access. The most frequent factors for changing the primary approach are challenging anatomical features such as carotid tortuosity or ostial stenosis, typ II or III arch, severe angulation or other vascular pathology like thoracic aortic aneurysms, severe thoracic aortic atherosclerosis, and Leriche syndrome. For type II or III arch, alternative transradial access is an option. Nevertheless, difficult access is associated with longer procedures, lower recanalization, and poorer outcome, and is consistent with our case series. ${ }^{6}$ Ribo et al. ${ }^{6}$ reported a recanalization rate (defined as $\mathrm{TICl}$ score $\geq 2 \mathrm{a}$ ) of $60.7 \%$ in patients ( $\mathrm{n}=123$ ) with difficult catheter access and lower favorable outcome of $13.6 \%$ (vs. 41.3\%) at 3 months. Recent studies with small samples showed even higher rates of successful reperfusion ( $\mathrm{mTICl}$ $\geq 2$ b) with $73 \%(n=11)$ and $100 \%(n=7)$, respectively. ${ }^{13,14}$ In our study, reperfusion (defined as $\mathrm{mTICI} \geq 2 \mathrm{~b}$ ) was achieved in $50 \%$ of patients. This might be due to prolonged time to reperfusion. Three patients presented as wake-up strokes, and the average groin to reperfusion time was 66 minutes. This was significantly longer than recent published results from our group (mean groin to reperfusion time, 34 and 41 minutes, respectively). ${ }^{11,15}$ The average time from femoral to carotid puncture was 33 minutes. Kaymaz et al. ${ }^{16}$ showed that supraaortic vessel tortuosity significantly influences internal carotid artery access time. A trend for lower successful recanalization rates with increasing internal carotid artery access time was found. ${ }^{16}$

One patient had regained excellent neurological function (mRS 5 at admission, mRS 1 at discharge and 90 days follow-up), whereas in the remaining four patients, endovascular procedure via transcarotid access had not improved initial neurological status. Overall outcomes in this groups are $14-18 \%$ and are consistent with our results. ${ }^{6,13}$ NIHSS was high at admission, and 50\% of patients didn't received concomitant intravenous thrombolysis. If no attempt at carotid 
access had been made, there would have been no patient with a favorable outcome.

Complications of a direct carotid artery puncture can be more severe in comparison to other approaches. Manipulating directly the carotid artery may increase the risk for peripheral intracranial embolism or carotid dissections, resulting in adverse events. Due to these factors, primary transcarotid approaches in elective procedures, such as cerebral angiography and stenting of the internal carotid artery, have been abandoned. Nevertheless, previous studies have reported a $2-7 \%$ rate of cervical hematoma after sheath withdrawal. ${ }^{10,17}$ Currently, there are no approved closure devices for the carotid approach. In our study, the Angio-Seal device (off label use) showed excellent success for carotid artery closure. No minor or major complications occurred. In addition, double platelet inhibition or intravenous thrombolysis treatment appeared to be safe with adequate hemostasis during and after closure.

Other reports are compatible with our results. ${ }^{12,17-19}$ Blanc et al. ${ }^{18}$ found complications (cervical hematoma, small aneurysm and puncture-related vasospasm, and thrombus formation) in six of 42 patients (14\%) encountered after performing manual compression of the access site. The Mynx device (vascular closure device; Cardinal Health, Santa Clara, CA, USA) did not show good success for closure of carotid arteries, and the use of Perclose ProGlide (Abbott Vascular; Santa Clara) or Exoseal (Cordis; Santa Clara) for this purpose has not been described. ${ }^{8}$ In a recent study performed by Wiesmann et al. ${ }^{7}$ open surgical access has been feasible but was associated with longer interventions (from the beginning of surgical procedure to the first DSA imaging via carotid access $42 \pm 19$ minutes) and this technique is much more complex to handle and is very dependent on optimal workflow and the availability of a vascular surgeon.

Ultrasound guidance and direct roadmap guidance are alternative techniques for carotid access. In our department, ultrasound is usually not available in the angiographic suite, and it is usually more difficult to handle (second operator). Therefore, we initiated the direct roadmap approach using a diagnostic catheter placed via a transfemoral access. In comparison with ultrasound guidance or open surgical access, our technique has increased radiation exposure, especially to the hands of the interventionalists, and therefore should only be used in selected patients and exceptional cases.

The major advantage of this technique is the continuous visibility of the complete internal carotid artery course during puncture and insertion of the sheath. After recanalization and removal of the cervical sheath, a control DSA can be performed with the maintained catheter in the proximal carotid artery placed via transfemoral access in order to detect possible vessel dissection or contrast extravasation.

\section{CONCLUSION}

A combined transfemoral/transcarotid access is feasible in selected patients if a transfemoral access is impossible or may significantly prolong procedure times. In our opinion, it's difficult to select these patients before the start of the interventional treatment. It's important that an experienced operator in the acute setting keeps an alternative carotid approach in mind and is prepared to switch access site after a defined period (for example 20 minutes) of unsuccessful transfemoral access to the carotid artery. The presented technical approach using direct roadmap guidance is simple and can be quickly adopted.

\section{REFERENCES}

1. Jo KW, Park SM, Kim SD, Kim SR, Baik MW, Kim YW. Is transradial cerebral angiography feasible and safe? A single center's experience. J Korean Neurosurg Soc 2010;47:332-337

2. Goland J, Doroszuk GF, Garbugino SL, Ypa MP. Transradial approach to treating endovascular cerebral aneurysms: case series and technical note. Surg Neurol Int 2017:8:73

3. Montorsi P, Galli S, Ravagnani PM, Tresoldi S, Teruzzi G, Caputi L, et al. Carotid artery stenting with proximal embolic protection via a transradial or transbrachial approach: pushing the boundaries of the technique while maintaining safety and efficacy. J Endovasc Ther 2016;23:549-560

4. Goyal M, Demchuk AM, Menon BK, Eesa M, Rempel JL, Thornton J, et al. Randomized assessment of rapid endovascular treatment of ischemic stroke. N Engl J Med 2015;372:1019-1030

5. Jovin TG, Chamorro A, Cobo E, de Miquel MA, Molina CA, Rovira $A$, et al. Thrombectomy within 8 hours after symptom onset in ischemic stroke. N Engl J Med 2015;372:2296-2306

6. Ribo M, Flores A, Rubiera M, Pagola J, Mendonca N, Rodriguez-Luna $D$, et al. Difficult catheter access to the occluded vessel during endovascular treatment of acute ischemic stroke is associated with worse clinical outcome. J Neurointerv Surg 2013;5 Suppl 1:i70-i73 
7. Wiesmann M, Kalder J, Reich A, Brockmann MA, Othman A, Greiner A, et al. Feasibility of combined surgical and endovascular carotid access for interventional treatment of ischemic stroke. J Neurointerv Surg 2016;8:571-575

8. Jadhav AP, Ribo M, Grandhi R, Linares G, Aghaebrahim A, Jovin TG, et al. Transcervical access in acute ischemic stroke. J Neurointerv Surg 2014;6:652-657

9. Castaño C, Remollo S, García MR, Hidalgo C, Hernández-Perez M, Ciorba M. Mechanical thrombectomy with "ADAPT" technique by transcervical access in acute ischemic stroke. Neuroradiol $J$ 2015;28:617-622

10. Alvarez B, Matas M, Ribo M, Maeso J, Yugueros X, Alvarez-Sabin J. Transcervical carotid stenting with flow reversal is a safe technique for high-risk patients older than 70 years. J Vasc Surg 2012;55:978-984

11. Maus V, Henkel S, Riabikin A, Riedel C, Behme D, Tsogkas I, et al The SAVE technique: large-scale experience for treatment of intracranial large vessel occlusions. Clin Neuroradiol 2018 Jul 19 [Epub]. https://doi.org/10.1007/s00062-018-0702-4

12. Mokin M, Snyder KV, Levy El, Hopkins LN, Siddiqui AH. Direct carotid artery puncture access for endovascular treatment of acute ischemic stroke: technical aspects, advantages, and limitations. J Neurolnterv Surg 2015;7:108-113
13. Roche A, Griffin E, Looby S, Brennan P, O'Hare A, Thornton J, et al. Direct carotid puncture for endovascular thrombectomy in acute ischemic stroke. J Neurointerv Surg 2019;11:647-652

14. Fjetland L, Roy S. Transcarotid endovascular thrombectomy for acute ischemic stroke. J Vasc Interv Radiol 2018;29:1006-1010

15. Psychogios MN, Behme D, Schregel K, Tsogkas I, Maier IL, Leyhe $J R$, et al. One-stop management of acute stroke patients: minimizing door-to-reperfusion times. Stroke 2017;48:3152-3155

16. Kaymaz ZO, Nikoubashman O, Brockmann MA, Wiesmann M, Brockmann C. Influence of carotid tortuosity on internal carotid artery access time in the treatment of acute ischemic stroke. Interv Neuroradiol 2017;23:583-588

17. Blanc R, Piotin M, Mounayer C, Spelle L, Moret J. Direct cervical arterial access for intracranial endovascular treatment. Neuroradiology 2006;48:925-929

18. Blanc R, Mounayer C, Piotin M, Sadik JC, Spelle L, Moret J. Hemostatic closure device after carotid puncture for stent and coil placement in an intracranial aneurysm: technical note. AJNR Am J Neuroradio/ 2002;23:978-981

19. Massiere B, von Ristow A, Cury JM, Gress M, Vescovi A, Pedron C, et al. Closure of carotid artery puncture site with a percutaneous device. Ann Vasc Surg 2009;23:256.e5-e7 